\title{
Alarm pheromone processing in the ant brain: an evolutionary perspective
}

\author{
Makoto Mizunami ${ }^{*}$, Nobuhiro Yamagata ${ }^{2}$ and Hiroshi Nishino ${ }^{3}$ \\ 1 Graduate School of Life Science, Hokkaido University, Sapporo, Japan \\ 2 Centre de Recherches sur la Cognition Animale, CNRS UMR 5169, Université Paul Sabatier, Toulouse Cedex 4, France \\ ${ }^{3}$ Research Institute for Electronic Science, Hokkaido University, Sapporo, Japan
}

\section{Edited by:}

Paul S. Katz, Georgia State University,

USA

\section{Reviewed by:}

Wulfila Gronenberg,

University of Arizona, USA

Manfred Schmidt,

Georgia State University, USA

${ }^{*}$ Correspondence:

Makoto Mizunami, Graduate School of Life Sciences, Hokkaido University,

Sapporo 060-0810, Japan.

e-mail:mizunami@sci.hokudai.ac.jp
Social insects exhibit sophisticated communication by means of pheromones, one example of which is the use of alarm pheromones to alert nestmates for colony defense. We review recent advances in the understanding of the processing of alarm pheromone information in the ant brain. We found that information about formic acid and $n$-undecane, alarm pheromone components, is processed in a set of specific glomeruli in the antennal lobe of the ant Camponotus obscuripes. Alarm pheromone information is then transmitted, via projection neurons (PNs), to the lateral horn and the calyces of the mushroom body of the protocerebrum. In the lateral horn, we found a specific area where terminal boutons of alarm pheromone-sensitive PNs are more densely distributed than in the rest of the lateral horn. Some neurons in the protocerebrum responded specifically to formic acid or $n$-undecane and they may participate in the control of behavioral responses to each pheromone component. Other neurons, especially those originating from the mushroom body lobe, responded also to non-pheromonal odors and may play roles in integration of pheromonal and non-pheromonal signals. We found that a class of neurons receive inputs in the lateral horn and the mushroom body lobe and terminate in a variety of premotor areas. These neurons may participate in the control of aggressive behavior, which is sensitized by alarm pheromones and is triggered by non-pheromonal sensory stimuli associated with a potential enemy. We propose that the alarm pheromone processing system has evolved by differentiation of a part of general odor processing system.

Keywords: pheromone, communication, antennal lobe, mushroom body, aggression, social insect, evolution

\section{INTRODUCTION}

Pheromones are potent signaling molecules that are fundamental for organizing a wide range of social behaviors in many vertebrates and invertebrates. In mammals, recent studies have demonstrated the importance of pheromones in influencing social behavior, but neural mechanisms of pheromone communication are poorly understood (Brennan and Kendrick, 2006; Brennan and Zufall, 2006). In insects, mechanisms by which sex pheromones are processed in the olfactory system have been extensively studied (Boeckh and Ernst, 1987; Kanzaki et al., 1994; Hildebrand and Shepherd, 1997), but little is known about how non-sexual, social pheromones are processed.

Eusocial insects such as ants, social bees, and termites have developed sophisticated communication systems by means of social pheromones (Blum, 1985; Hölldobler, 1995; Vander Meer and Alonso, 1998). Functional division into reproductive and sterile castes, cooperation in defending the nest, rearing the young and gathering food, which are hallmarks of eusociality, are all regulated by communication by means of various kinds of pheromones. Unusual sophistication of pheromone communication in eusocial insects is evidenced by the fact that they possess a diverse array of exocrine glands devoted to social communication, 39 such glands existing in formicine ants and 21 existing in honey bees (Billen, 1994). Therefore, social insects, especially ants, provide excellent materials to study neural mechanisms underlying the processing of social pheromones.
This review deals with alarm pheromone processing in the ant brain, but we first briefly summarize current knowledge of the processing of general environmental odor and sex-pheromone information in the insect brain, because such knowledge provides a substantial basis for understanding alarm pheromone processing. In insects, olfactory (volatile) molecules are received by olfactory receptor neurons (RNs) housed in sensilla on the antennae. Axons of RNs project to the primary olfactory center, the antennal lobe (a lob in Figures 1 and 2A), which is the functional analog of the olfactory bulb in mammals (Hildebrand and Shepherd, 1997). In fruit-flies, the axons of RNs that express the same type of olfactory receptor gene converge, in principle, on a single glomerulus in the antennal lobe, and the glomeruli thus serve as functional units (Couto et al., 2005; Vosshall and Stocker, 2007). In each glomerulus, a number of RNs make synaptic connections with a small number of projection neurons (PNs), output neurons of the antennal lobe, and a number of local interneurons. Axons of PNs pass through the antenno-cerebral tracts and terminate in the lateral horn and the calyces of the mushroom body of the protocerebrum (l ho and ca in Figures 1 and 2A). In the protocerebrum, signals of various sensory systems are integrated to lead to behavioral decision (Strausfeld, 1976; Okada et al., 2003).

Receptor neurons that respond to general environmental odors are characterized by their relatively wide receptive ranges in relation to the kinds of chemicals. Thus, information about a 


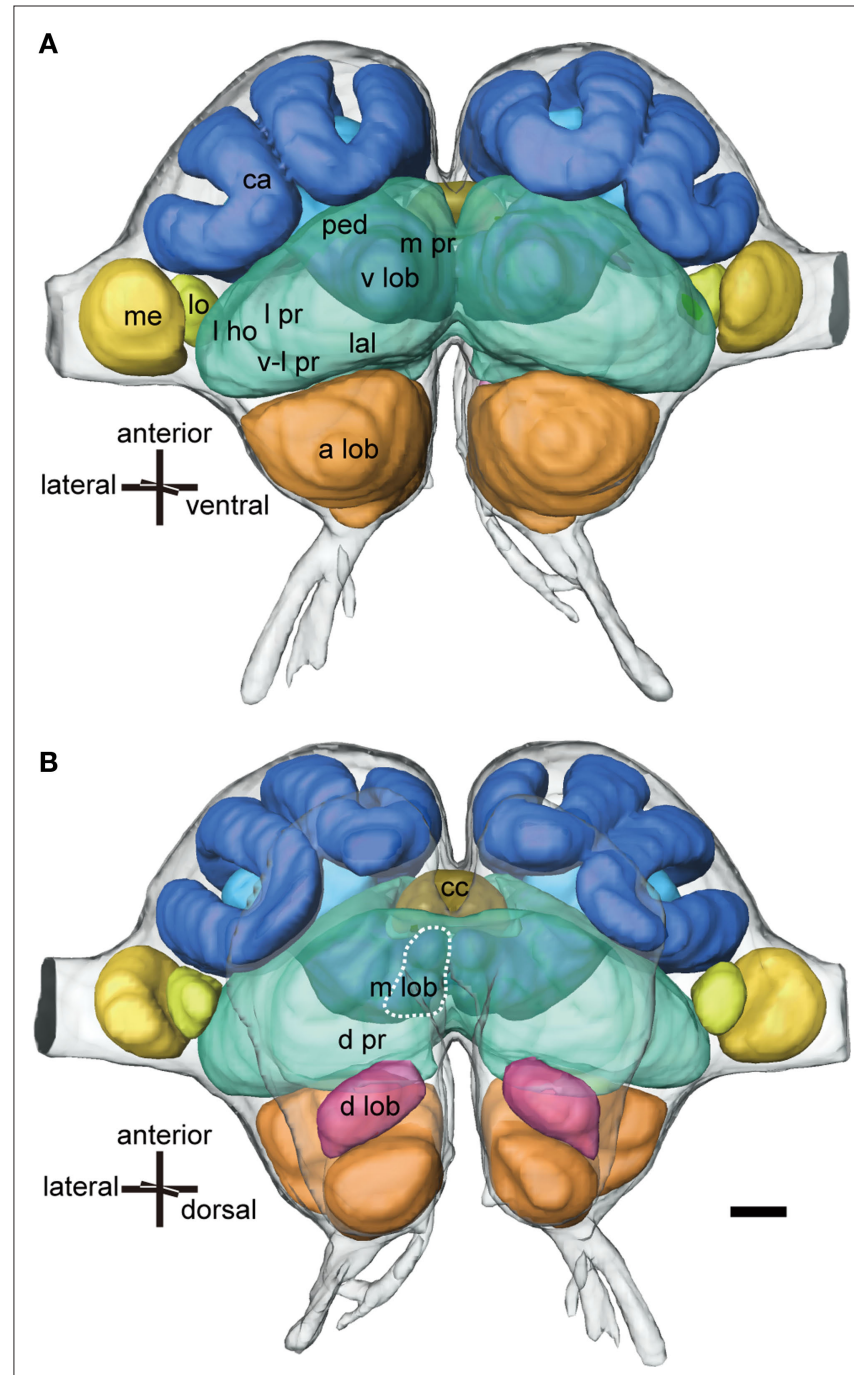

FIGURE 1 |Three-dimensional reconstructions of major neuropils of the ant brain viewed ventrally (A) and dorsally (B). The protocerebrum (green) consists of the mushroom body, the lateral PR (I pr) that includes the lateral horn (I ho), the ventro-lateral PR ( $\mathrm{v}-\mathrm{l}$ pr), the medial and dorsal PR ( $\mathrm{m}$ pr and $\mathrm{d}$ pr), the lateral accessory lobe (lal) and the central complex (cc: khaki). The mushroom body consists of calyces (ca: blue), the pedunculus (ped: light blue) and the vertical and medial lobes ( $\mathrm{v}$ lob, $\mathrm{m}$ lob: light blue, broken line). The medulla (med: yellow) and the lobula (lo: light yellow) are the second and third optic neuropils. The deutocerebrum consists of the antennal lobe (a lob: orange) and the dorsal lobe (d lob: magenta). Scale bar $=100 \mu \mathrm{m}$. Modified from Yamagata et al. (2007).

general environmental odor is coded as an activity pattern among a number of RNs and glomeruli (Joerges et al., 1997; de Bruyne et al., 2001). This combinatory, across-fiber pattern coding enables insects to discriminate an enormous number of different odors (e.g., in honey bees: Vareschi, 1971) by using a limited number of types of receptor molecules and RNs. In addition to the combinatory code, synchronous firing of PNs is reported to play roles in discriminating similar odors (Stopfer et al., 1997). The combinatory code is decoded in higher olfactory centers. Both the lateral horn and the mushroom body may participate in such decoding.

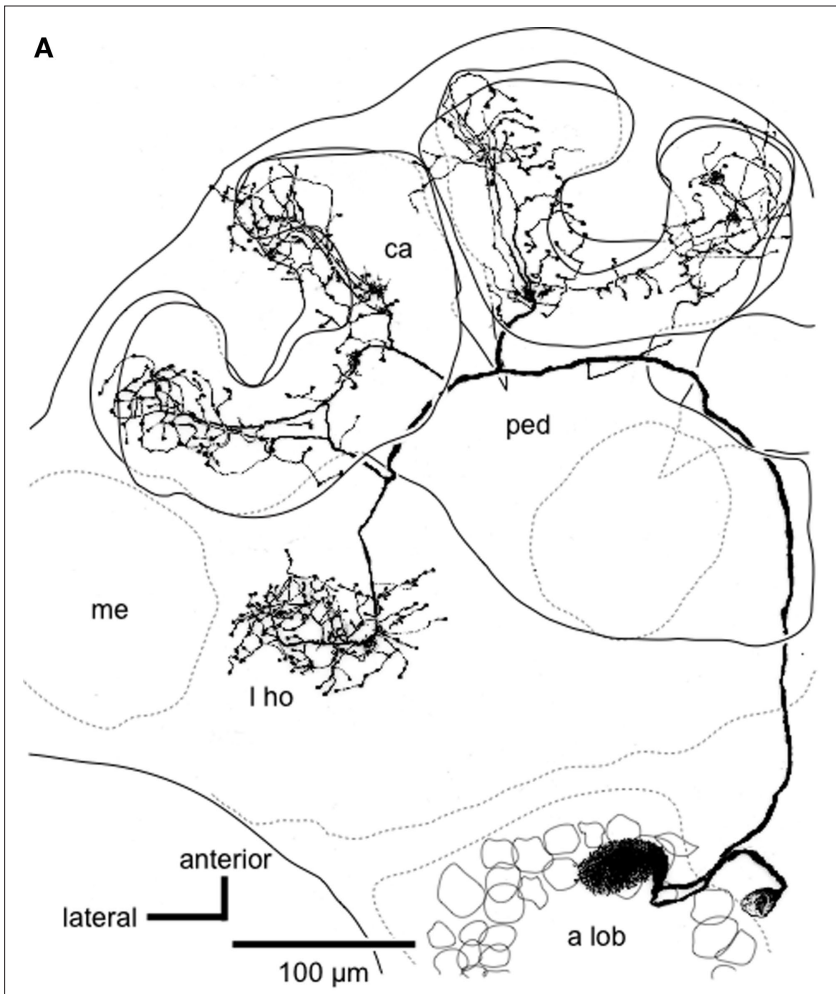

B

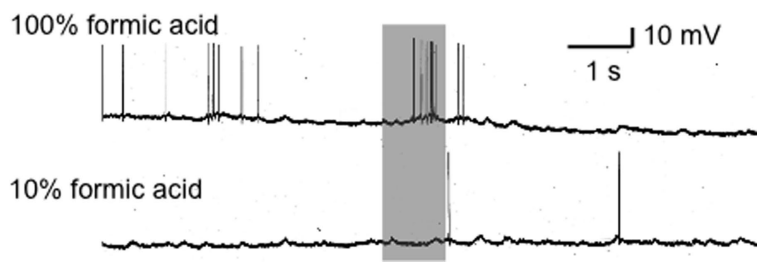

FIGURE 2 |An alarm pheromone-sensitive uniglomerular PN.

(A) Reconstruction of an alarm pheromone-sensitive PN from confocal sections viewed ventrally. The soma is located at the medial cell cluster of the deutocerebrum. The dendritic arbors cover the AS1 glomerulus in the antennal lobe (a lob). The axon ascends through the medial anteno-cerebral tract and terminates in the lip region of the calyces (ca) and the lateral horn (I ho). me: medulla, ped: pedunculus. (B) Responses of the PN to formic acid. 10\% formic acid indicates that it was diluted to $1 / 10$ by distilled water. Modified from Yamagata et al. (2006).

In contrast to information about general environmental odors, sex pheromone information is coded in specialized neural channels. Sex pheromones are sensed by highly specific RNs with narrow molecular receptive ranges (cockroaches: Boeckh and Ernst, 1987; moths: Kanzaki et al., 1994; Hildebrand and Shepherd, 1997). Axons of RNs project to specialized glomeruli called macroglomerular complex. PNs originating from the macroglomerular complex terminate in a specific region of the lateral horn, which is, in most part, segregated out from the region to receive termination of PNs conveying information about general environmental odors (in cockroaches: Nishino et al., 2003; in moths: Kanzaki et al., 2003; Seki et al., 2005; in fruit-flies: Jefferis et al., 2007). This labeled line code enables insects to rapidly and reliably respond to sex pheromones, because the signal can be used for behavioral control without passing through central decoding circuitry. A critical 
question to be addressed in examining non-sexual social pheromone processing is, therefore, how social pheromone processing is characterized in comparison with labeled line coding for sex pheromones and combinatory, across-fiber pattern coding for general environmental odors.

Alarm pheromones play essential roles for colony defense in social insects (Blum, 1985), and we have examined processing of alarm pheromones in the brain of the worker of the carpenter ant Camponotus obscuripes. We have anatomically and physiologically characterized 86 brain neurons that responded to alarm pheromone components by means of intracellular recording and staining techniques (Yamagata et al., 2005, 2006, 2007; Yamagata and Mizunami, 2010). Here we integrate the results of our study with those of other studies to discuss about design principles of the alarm pheromone processing system and its evolution.

\section{BEHAVIORAL RESPONSES TO ALARM PHEROMONES OF THE ANT}

Upon facing a potential danger such as a predator, workers of ants, or social bees secrete alarm pheromones to warn their conspecifics about danger (Blum, 1985). Although behavioral responses of ant workers to alarm pheromones in the context of colony defense are highly complicated and diverse, they can be classified into three sequential patterns: (1) initial response to pheromones, (2) alarm behavior, and (3) aggressive behavior against a potential enemy, which is sensitized by alarm pheromones and is triggered by sensory stimuli associated with the potential enemy (Blum, 1985; Vander Meer and Alonso, 1998). In most cases, alarm pheromones alone do not trigger aggressive behavior.

The above general scheme is useful to understand behavioral responses of workers of the carpenter ant C. obscuripes to alarm pheromones. In this species, products of the poison gland and Dufour's gland act as alarm pheromone, the former being formic acid and a predominant component of the latter being $n$-undecane (Fujiwara-Tsujii et al., 2006). In the initial response to alarm pheromones, workers that have sensed pheromones reflexively stop their locomotion, swing their antennae, and then come to an alerted posture. Thereafter, they exhibit alarm behavior of different intensities, which usually includes locomotion of increased speed with alerted posture. Workers then exhibit a specific behavioral reaction to each alarm pheromone component, if it is presented alone, namely, avoidance of the source of formic acid and attraction toward the source of $n$-undecane (Fujiwara-Tsujii et al., 2006). Therefore, the ratio of formic acid and $n$-undecane influences the alarm behavior in this species.

Alarm pheromones sensitize aggressive behavior against a potential enemy in workers of C. obscuripes. Aggressive behavior in ants is triggered by olfactory, visual or tactile stimuli associated with the enemy (Blum, 1985; Vander Meer and Alonso, 1998) and is also influenced by many other external and internal factors (Blum, 1985; Vander Meer and Alonso, 1998), including the state of the path integrator that tells the ant how far it is away from the nest (Knaden and Wehner, 2004). In workers of honey bees, effects of age, strains (inherited factors) and expression of a set of genes in the brain on behavioral responses to alarm pheromones have been examined (Hunt, 2007; Alaux et al., 2009).

\section{RNs FOR ALARM PHEROMONES}

Physiological characterization of RNs on antennae that respond to alarm pheromone components has been limited to the black garden ant Lasius fuliginosus (Dumpert, 1972). Dumpert (1972) characterized response profiles of RNs housed in trichoid sensilla, a major type of olfactory sensilla on ant antennae, and classified them into 10 types. Among them, one type of RNs specifically responded to $n$-undecane, alarm pheromone of this species, namely, the sensitivity to $n$-undecane was at least three orders of magnitude higher than that to structurally related odorants such as $n$-decane, 2-methylundecane, and 2-methyl-1-undecane. Another type responded to formic acid. This type also responded to various kinds of carboxylic acids, including acetic acid, propionic acid, and heptanoic acid. Thus, formic acid is recognized not by formic acid-specific receptors but by carboxylic acid receptors in this species.

\section{SEXUAL DIMORPHISM IN THE ANT ANTENNAL LOBE}

In ants, the worker and the queen possess a larger number of glomeruli than males, indicating that females possess additional types of receptor neurons. In the carpenter ant C. japonicus, the worker and the queen each have ca. 430 glomeruli, whereas the male has ca. 215 glomeruli (Nakanishi et al., 2010). In C. floridanus, the worker and queen each have ca. 434 glomeruli, whereas the male has only ca. 258 glomeruli (Zube et al., 2008; Zube and Rössler, 2008). Recent studies suggest that the difference in number of glomeruli is, in large part, due to the presence or absence of glomeruli involved in social pheromone processing. Glomeruli in ants have been classified into 7 clusters (T1-T7) according to the tracts of RN axons (Nishikawa et al., 2008; Zube et al., 2008; Mysore et al., 2009), and ca. 140 T6 glomeruli present in females are absent in males (Nakanishi et al., 2010). T6 glomeruli very likely receive axonal projection of RNs housed in basiconic sensilla (Nakanishi et al., 2009, 2010), which respond to cuticular hydrocarbon blends and function in discriminating nestmates and non-nestmates (Ozaki et al., 2005).

The number of glomeruli in female ants is, in general, larger than that reported in any other insects, including social bees: e.g., the number of glomeruli is $\sim 50$ in the male and female of fruit-flies (Couto et al., 2005), 63 in both sexes of hawk moths (Rospars and Hildebrand, 2000), 174 in the worker and ca. 116 in the drone of honey bees (Nishino et al., 2009) and 205 in both sexes of American cockroaches (Watanabe et al., 2010). Locusts have $\sim 1,000$ glomerulus-like structures, but they should be regarded as subunits of glomeruli (Galizia and Rössler, 2010). The observation indicates that a larger number of olfactory receptor types have been evolved in ants than in other insects, including honey bees, which may be an adaptation to underground lifestyle relying heavily on olfaction rather than vision and also to allow for unusually sophisticated social communication.

\section{ALARM PHEROMONE PROCESSING IN THE ANTENNAL LOBE}

By use of intracellular recording and staining techniques, we have characterized responses of PNs of the antennal lobe to alarm pheromone components (formic acid and $n$-undecane) and to several non-pheromonal odors (such as vanilla, peppermint, banana, apple, and peach) in workers of C. obscuripes (Yamagata et al., 2006). We refer to the neurons that responded to formic acid and/or $n$-undecane 
as pheromone-sensitive neurons: Some of these neurons specifically responded to formic acid and/or $n$-undecane and others responded also to non-pheromonal odors. Among 23 alarm pheromone-sensitive $\mathrm{PNs}$ recorded and stained, 8 were uniglomerular PNs with dendrites in one glomerulus, and the remaining 15 were multiglomerular PNs with dendrites in a large number of glomeruli (for multiglomerular PNs of other species of insects; see Galizia and Rössler, 2010). All alarm pheromone-sensitive uniglomerular PNs had dendrites in one of five 'AS (alarm pheromone-sensitive)' glomeruli that form a cluster in the dorsalmost part of the antennal lobe (Figures 2 and 3). All alarm pheromone-sensitive multiglomerular PNs had dendrites in some of the AS glomeruli as well as in glomeruli in the anterodorsal area of the antennal lobe.

We found functional subdivision among five AS glomeruli (Figure 3). PNs with dendrites in AS1 $(n=2$, an example of which is shown in Figure 2$)$ or AS2 $(n=2)$ responded to formic acid but did not respond to $n$-undecane or any other odors tested, whereas an AS3 PN $(n=1)$ specifically responded to $n$-undecane. AS4 PNs $(n=2)$ or an AS5 PN $(n=1)$ responded to both pheromone components: The latter did not respond to any non-pheromonal odors but the former responded to one or a few non-pheromonal odors (Figure 3). Each of the AS1-AS3 glomeruli is likely to receive axon terminals of RNs that specifically respond to formic acid or

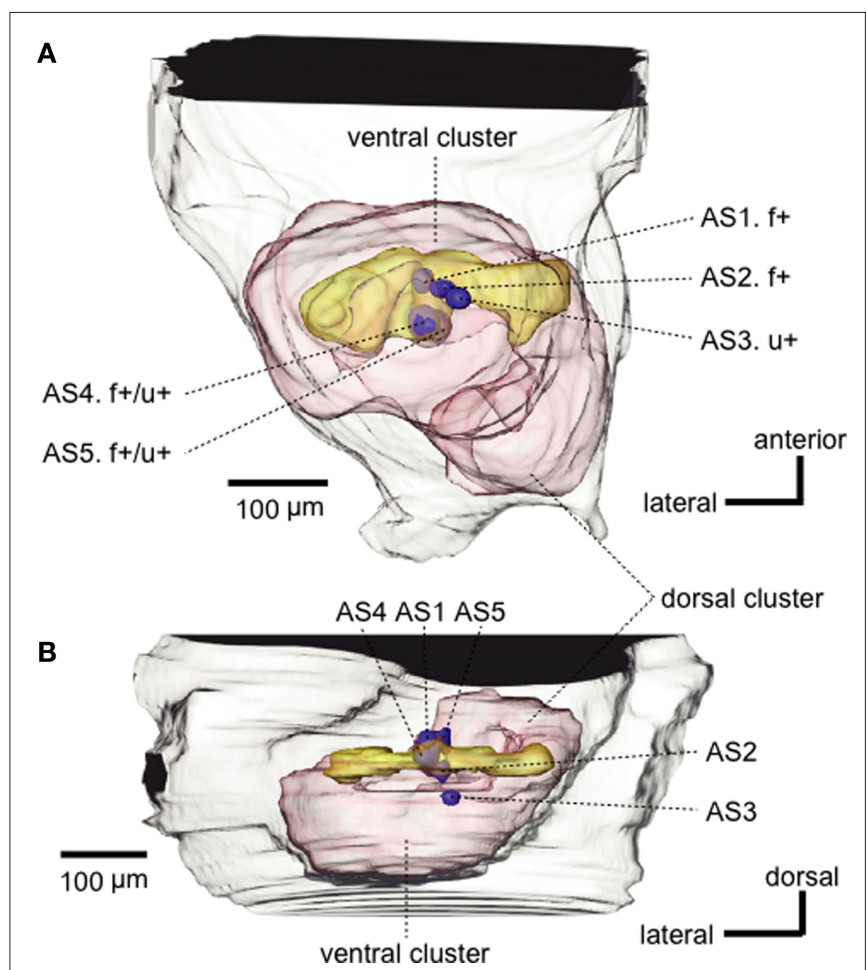

FIGURE 3 |Three-dimensional reconstruction of the antennal lobe of the ant, viewed ventrally (A) and horizontally (B). Five glomeruli (blue spheroids, AS1-AS5) from which alarm pheromone-sensitive uniglomerular PNs originated are mapped into the reconstructed antennal lobe (red). $f+$ and $\mathrm{u}+$ indicate that the PN originating from that glomerulus was sensitive to formic acid and $n$-undecane, respectively. Alarm pheromone-sensitive multiglomerular PNs had dendrites in some of the AS glomeruli as well as in a number of glomeruli in the dorsalmost part (yellow) of the ventral glomerular cluster. Modified from Yamagata et al. (2006). $n$-undecane, but it is unclear whether the AS4 and AS5 glomerulus receives axon terminals of both types of RNs or whether the observed responses are due to interactions among neighboring glomeruli, via local interneurons. It is also unknown whether non-pheromonal responses of AS4 PNs reflect relatively low specificity of RNs or are due to interactions via local interneurons. In contrast to pheromone-sensitive uniglomerular PNs, most of the pheromone-sensitive multi-glomerular PNs responded also to non-pheromonal odors, reflecting their dendritic morphology. The exact number of AS glomeruli and detailed response characteristics of their uniglomerular PNs remain to be clarified.

\section{POSSIBLE FUNCTIONAL SEGREGATION BETWEEN PHEROMONE-SENSITIVE UNIGLOMERULAR AND MULTIGLOMERULAR PNs}

Uniglomerular and multiglomerular PNs differ not only in their dendritic morphologies and olfactory responses but also in termination areas of their axons in the protocerebrum. Axons of uniglomerular PNs terminate in the lateral horn and the calyces of the mushroom body (Figure 2A); the latter is known to participate in multisensory integration and several forms of learning and memory (honey bees: Erber et al., 1980; Menzel and Giurfa, 2001; cockroaches: Mizunami et al., 1998a,b; fruit-flies: Heisenberg, 2003). In contrast, axons of multiglomerular PNs terminate in the lateral horn, lateral network of the lateral protocerebrum, ring neuropil around the vertical lobe of the mushroom body and dorsal protocerebrum (see Figure 1), the latter three areas being sites where many descending brain neurons originate to supply thoracic motor centers (cockroaches: Okada et al., 2003). We have deduced that pheromone-sensitive multiglomerular PNs provide a rapid sensorimotor pathway for termination of ongoing behavior and preparation for alarm behavior, whereas uniglomerular PNs comprise a major pathway to convey detailed signals about alarm pheromones for the control of pheromone-induced alarm behavior and pheromone-sensitized aggressive behavior (Yamagata et al., 2007), as will be discussed in Section 'Alarm pheromone processing in the mushroom body and its downstream protocerebral areas'.

\section{FUNCTIONAL ORGANIZATION AND POSSIBLE EVOLUTION OF THE ALARM PHEROMONE PROCESSING SYSTEM IN THE ANT BRAIN}

Our findings briefly summarized above indicate that alarm pheromone components are coded in a labeled line system in ants, as are sex pheromone components in moths, cockroaches, and honey bees. It should be pointed out, however, that the specificity of the neurons to code alarm pheromones is not as high as that for coding sex pheromones; that is, some AS glomeruli responded to both alarm pheromone components and one of them even responded to a few non-pheromonal odors. This is in contrast to the fact that each subdivision of the macroglomerular complex specifically responds to each component of the sex pheromone in moths (Kanzaki et al., 1994; Hildebrand and Shepherd, 1997) and cockroaches (Boeckh and Ernst, 1987).

Labeled line coding of social pheromone information has also been suggested in leaf-cutting ants. The antennal lobe of large workers of leaf-cutting ants contains an enlarged glomerulus (Kleineidam et al., 2005) and recent calcium imaging studies have shown that it 
and its neighboring glomerulus respond to different components of trail pheromones (Kuebler et al., 2010). Detailed olfactory responses of these glomeruli, however, have not been reported.

In contrast to those studies, however, some other studies on ants suggest across-glomeruli activity pattern coding of components of the alarm pheromone and other social pheromones. Calcium imaging studies of neural activities of glomeruli at the ventral surface of the antennal lobe have shown that the glomeruli that responded to $n$-undecane or other social pheromones also responded to nonpheromonal odors in carpenter ants (Camponotus rufipes: Galizia et al., 1999a; C. floridanus: Zube et al., 2008). Thus, it is concluded that alarm pheromone components are coded by across-glomeruli activity patterns. Similar combinational representation of a major alarm pheromone component (isoamyl acetate) with non-pheromonal odors has been found in calcium imaging studies of glomeruli at the ventral surface of the antennal lobe in honey bees (Galizia et al., 1999b; Sachse et al., 1999). These studies, however, have dealt only with glomeruli at the ventral surface of the antennal lobe, due to limited accessibility of optical recording techniques to deeper glomeruli. Conversely, we have not yet encountered alarm pheromone-sensitive uniglomerular PNs with dendrites in glomeruli at the ventral surface of the antennal lobe in C. obscuripes.

These different observations in different studies on ants can be reconciled if alarm pheromones are coded in a labeled line system at the dorsal part of the antennal lobe and in an across-fiber pattern coding system at the ventral part of the antennal lobe (Figure 4). Such dual coding system may be advantageous because it would allow ants to use an alarm pheromone component for recognizing both alarm pheromones and general environmental odors, depending on the behavioral context that ants receive the odor, because the alarm pheromone component may also be a component of food or other environmental odors.
Notably, dual coding of overlapping sets of odors have been suggested in honey bees. Uniglomerular PNs in the lateral or medial antenno-cerebral tract, each originating from glomeruli at the ventral or dorsal part of the antennal lobe (Kirschner et al., 2006), exhibit highly overlapping response profiles to a number of odors. Thus, they are thought to transmit different aspects of information (such as odor quality versus odor concentration or fast odor fluctuations versus slow odor changes) for overlapping sets of odors (Müller et al., 2002; Krofczik et al., 2008; Yamagata et al., 2009; Galizia and Rössler, 2010). The labeled line system for social pheromones like alarm pheromones may have evolved by differentiation of a part of such duplicative pattern coding systems to facilitate rapid and reliable behavioral responses to social pheromones.

It remains to be studied whether our finding that alarm pheromones are processed in specific glomeruli is applicable to other social pheromones in ants, since there is no a priori reason to speculate that all social pheromones are coded in specific neural channels. Moreover, it needs to be clarified whether the finding in ants that some of the social pheromones are processed in specific glomeruli is applicable to honey bees. Honey bees have a much smaller number of glomeruli than that in ants (see Sexual dimorphism in the ant antennal lobe) and no glomeruli specifically processing non-sexual social pheromones have been reported (Sandoz et al., 2007).

\section{ALARM PHEROMONE PROCESSING IN THE LATERAL HORN}

Are there areas to specifically process alarm pheromone information in the lateral horn of ants? In order to examine this question, we studied distributions of terminal boutons of alarm pheromone-sensitive and pheromone-insensitive PNs in the lateral horn of the worker of C. obscuripes (Yamagata and Mizunami, 2010). We found no region to specifically receive alarm pheromone

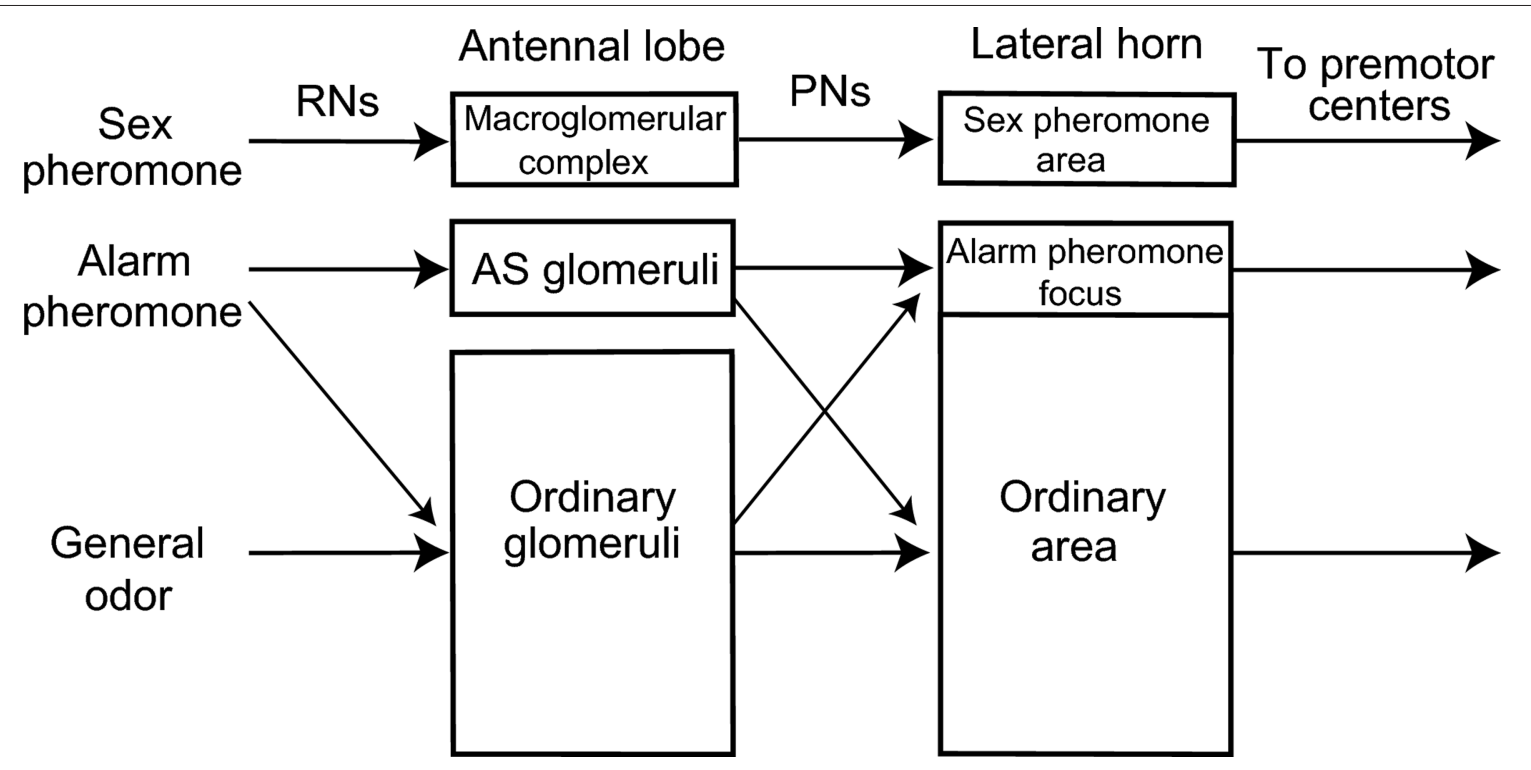

FIGURE 4 | A model of alarm pheromone processing system in the ant brain. Organization of the alarm pheromone processing system in the worker of the ant C. obscuripes is schematically compared with the general odor processing system and sex-pheromone processing system of other insects, such as moths, cockroaches, honeybees and fruit-flies. There is segregated, yet partially overlapped, representation of alarm pheromone information in the primary (antennal lobe) and a secondary (lateral horn) olfactory center. This is in contrast to segregated representations of sex-pheromone information. For details, see text. 
information: Terminal boutons of pheromone-sensitive PNs were widely distributed in the lateral horn and all regions that received alarm pheromone information also received non-pheromonal information. This is in contrast to the findings that sex pheromone information is represented in a region almost segregated from regions to process other odors in cockroaches (Nishino et al., 2003), moths (Kanzaki et al., 2003; Seki et al., 2005), and fruitflies (Jefferis et al., 2007). However, we found that an area in the antero-medial region of the lateral horn terminal boutons of pheromone-sensitive PNs are significantly denser than in the rest of the lateral horn. The density of terminal boutons of pheromone-insensitive PNs did not differ in this region and in the rest of the lateral horn. We refer to this region as the alarm pheromone focus (Figure 4). We also found that most of a major type of alarm pheromone sensitive efferent (output) neurons of the lateral horn, called wide-field protocerebral neurons (Yamagata et al., 2007), extended dendritic branches in this region, suggesting specialization of this region for alarm pheromone processing. The observation suggests that processing of alarm pheromones differs from that of general environmental odors in the lateral horn, because it is unlikely that each of thousands of general environmental odors, such as peppermint or vanilla, has its own specific focus in the lateral horn. It should also be noted that this area receives non-pheromonal odor and may play roles in integrating alarm pheromonal information with non-pheromonal information, in addition to process alarm pheromones and send the information to a type of output neurons.

The lateral horn area outside the alarm pheromone focus, which we refer to as the ordinary area, mainly receives terminal boutons from PNs responding to general odors, but it also receives terminal boutons of pheromone-sensitive PNs (Figure 4). We speculate that alarm pheromone information provides contextual signals for the processing of general environmental odors in this part of the lateral horn.

We observed no obvious differences in the distribution of terminal boutons between PNs that are sensitive to formic acid $(n=7)$ and those sensitive to $n$-undecane $(n=3)$. This is in contrast to the fact that different sex pheromone components are represented in partially segregated areas in the lateral horn of the moth: uniglomerular PNs conveying signals of different components of sex pheromones terminate in partially segregated areas in the lateral horn (Seki et al., 2005). PNs often exhibit different responses to formic acid and $n$-undecane (see Figure 4, Yamagata et al., 2006), which should be the basis for different behavioral responses to them.

\section{ALARM PHEROMONE PROCESSING IN THE MUSHROOM BODY AND ITS DOWNSTREAM PROTOCEREBRAL AREAS}

We have anatomically and physiologically characterized 63 neurons that responded to formic acids and/or $n$-undecane in the protocerebrum in workers of $C$. osbcuripes (Yamagata et al., 2007). Most of these neurons had dendrites in the lobes or pedunculus of the mushroom body, the lateral horn, and/ or the medial protocerebrum (see Figure 1), the last area being a major termination area of efferent (output) neurons of the lobes or the pedunculus of the mushroom body. Some of these neurons responded specifically to formic acid ( $n=12,19 \%)$ or $n$-undecane $(n=1,2 \%)$. These neurons are likely to participate in the control of specific behavioral response to formic acid or $n$-undecane. Other neurons $(n=14,22 \%)$ responded to formic acid and $n$-undecane but not to non-pheromonal odors tested, and the remaining neurons $(n=36,57 \%)$ responded also to nonpheromonal odors, some of which responded also to visual and/ or tactile stimuli. Most (16 out of $18,89 \%$ ) of the pheromonesensitive output neurons of the lobe of the mushroom body exhibited responses to non-pheromonal odors. These neurons are likely to play roles in the integration of pheromonal signals with non-pheromonal signals.

We have proposed a general scheme for neural pathways for alarm pheromone processing in the ant brain, on the basis of observations of 63 alarm pheromone-sensitive protocerebral neurons (Figure 5, Yamagata et al., 2007). This scheme shows that both neural pathways for alarm pheromone processing and behavioral responses to alarm pheromones are diverse and highly complicated, and there are no simple ways to relate particular neural pathways to particular behavior. This scheme, however, provides a starting point for considering such relationships. In this scheme, we suggest two sensori-motor pathways for pheromone processing in relation to two separate termination areas of uniglomerular PNs. In the first pathway, the lateral horn (1 ho) intervenes between the antennal lobe and premotor areas of the protocerebrum, and in the second pathway, the mushroom body $(\mathrm{mb})$ intervenes between them. The neural pathway involving the lateral horn is implicated in some stereotyped behaviors such as innate avoidance of particular odors (in fruit-flies: Heimbeck et al., 2001; Suh et al., 2004), while the mushroom body and its downstream protocerebral areas are implicated in multisensory integration, learning and memory, and the processing of contextual signals (Honey bees: Erber et al., 1980; Menzel and Giurfa, 2001; cockroaches: Mizunami et al., 1998a,b; Li and Strausfeld, 1999; Okada et al., 1999; fruit-flies: Heisenberg, 2003). We propose that the pathway involving the lateral horn plays major roles in mediating alarm behavior, which is more or less stereotyped, while the pathway involving the mushroom body plays major roles in mediating aggressive behavior, which is controlled by contextual information like the presence of alarm pheromones (Blum, 1985). In fruit-flies, learning and memory play important roles in the control of aggressive behavior (Yurkovic et al., 2006) and this may be applicable to ants. Signals from these two pathways converge at various premotor centers: pheromone-sensitive neurons with dendrites in the lateral horn and those with dendrites in the lobes and/or the pedunculus of the mushroom body converge at various protocerebral areas including the medial ( $\mathrm{m}$ pr), lateral (l pr) and dorsal (d pr) protocerebrum and lateral accessory lobe (lal).

Notably, we found a class of neurons $(n=12)$ that receives input in the lateral horn, the vertical lobe of the mushroom body and medial protocerebrum (Figure 6A). Axons of these neurons terminate in a variety of premotor areas, including the lateral accessory lobe and dorsal protocerebrum, from which descending neurons originate to supply thoracic motor centers. In some of these neurons, the axons further descend toward the subesophageal ganglion. 


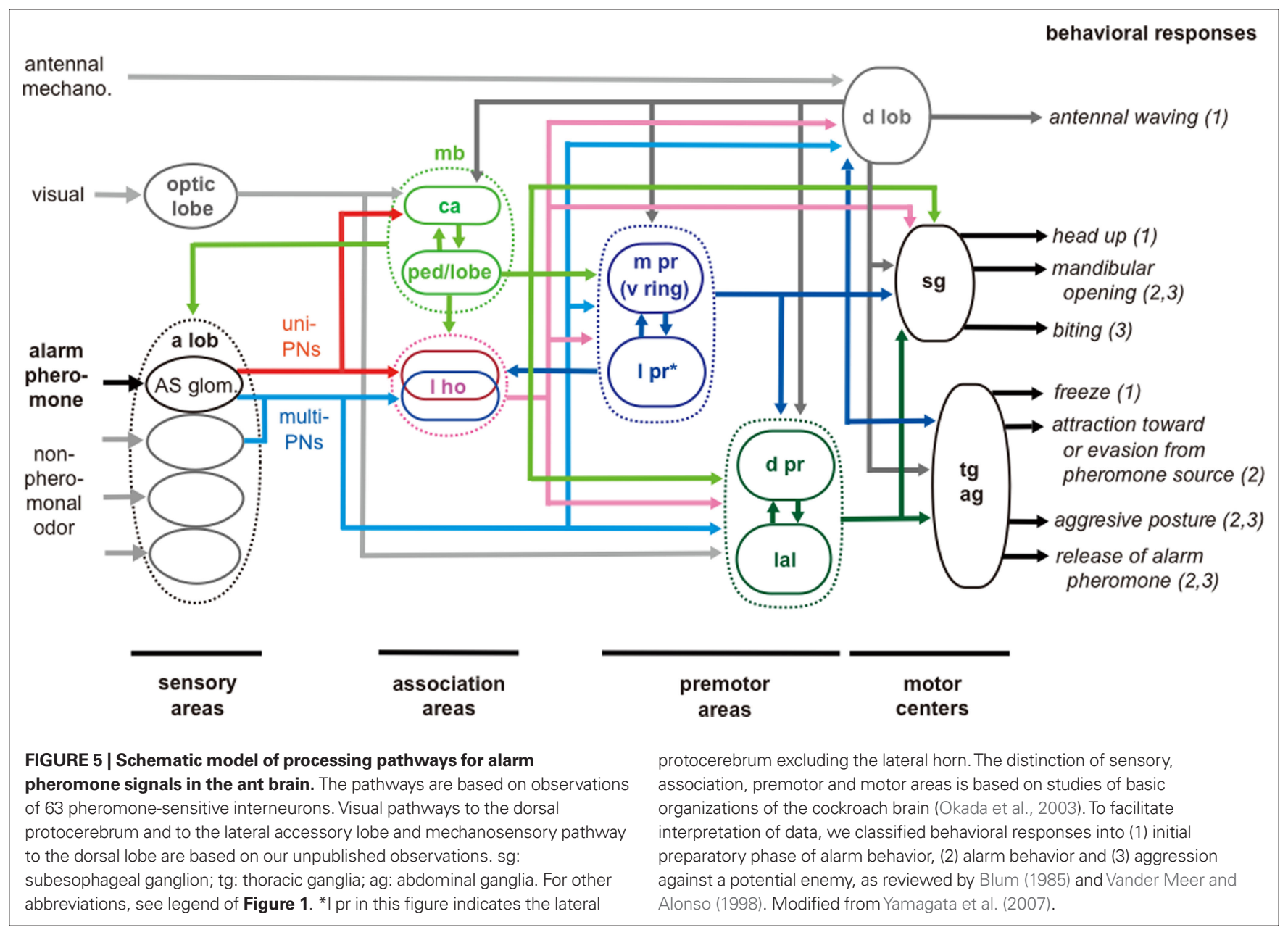

Most of these neurons ( $n=11,92 \%)$ exhibited integration of pheromonal and non-pheromonal information (Figure 6B), which is essential for the control of aggressive behavior. These neurons are likely to participate in the control of aggressive behavior against a potential enemy.

One pheromone-sensitive descending protocerebral neuron exhibited long-lasting spike activity (for $4 \mathrm{~s}$ ) in response to alarm pheromone stimulation (Yamagata et al., 2007). This neuron had dendrites in the lateral accessory lobe and the medial protocerebrum. Similar long-lasting spike activities have been reported from sex-pheromone-sensitive descending protocerebral neurons with dendrites in the lateral accessory lobe in the silkmoth (Kanzaki et al., 1994). In the silkmoth, these neurons have been shown to participate in the control of orientation behavior toward the source of the sex pheromone (Kanzaki et al., 1994).

Therefore, alarm pheromone-sensitive descending neurons may participate in the control of orientation behavior toward or away from the sources of the pheromone.

\section{FUTURE PERSPECTIVES}

Communication by means of pheromones is highly sophisticated in social insects, especially in ants, and our studies provide a starting point for elucidating brain mechanisms underlying pheromone communication in ants. Future studies should include better physiological and anatomical characterization of receptor neurons responding to alarm pheromones. Moreover, our proposal that alarm pheromone information is dually coded in a labeled line system and in an across-fiber-pattern coding system (Figure 4) has not yet been demonstrated in any species of insects, and studies are needed to characterize glomeruli that receive terminations of axons of pheromone-sensitive RNs to evaluate the validity of our proposal. In addition, comparison of olfactory systems between solitary wasps and eusocial hymenoptera should also provide critical information to understand evolution of social pheromone processing systems in eusocial hymenoptera. Another interesting subject is to examine possible roles of wide-field protocerebral neurons in the control of aggressive behavior, because neural and molecular mechanisms underlying the control of aggressive behavior are major topics in current neurobiology (Breed et al., 2004; Alaux et al., 2009; Wang and Anderson, 2010).

Insects have very small brains consisting of $10^{6}$ neurons or less, yet they exhibit diverse and sophisticated behaviors, examples of which are alarm behavior and colony defense behavior of ants discussed in this article. Elucidation of design principles of insect 'microbrains' (Mizunami et al., 1999, 2004; see also Chittka and 


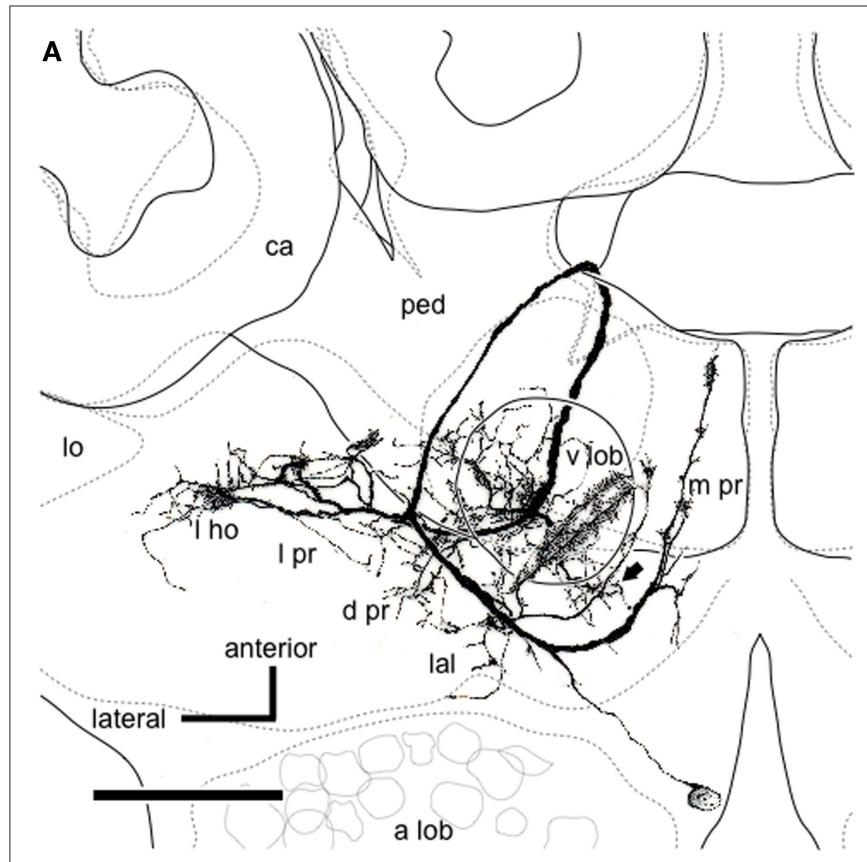

FIGURE 6 | Pheromone-sensitive wide-field protocerebral neuron.

(A) Reconstruction of the neuron from confocal sections viewed ventrally. The soma is located at the boundary between the protocerebrum and the antennal lobe (a lob). The dendrites cover the vertical lobe ( $\mathrm{llob}$ ), the medial protocerebrum ( $\mathrm{m} \mathrm{pr}$ ) and a part of the lateral horn (I ho) and the lateral accessory lobe (lal). The axon takes a circular route around the pedunculus (ped) and

\section{B}

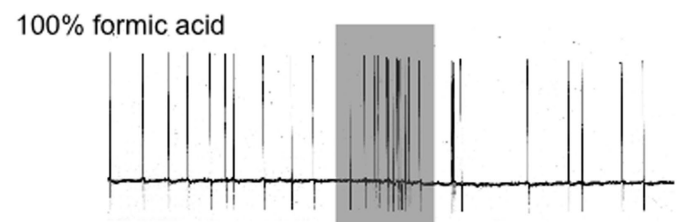

1-hexanol

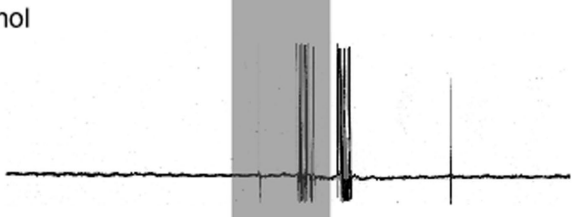

banana

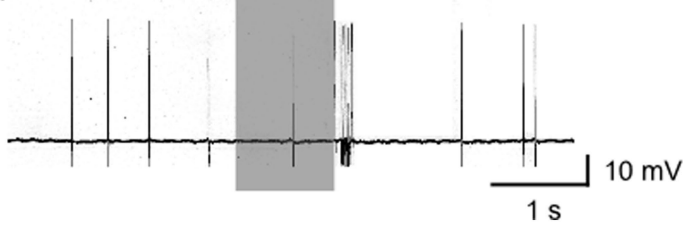

Niven, 2009) should provide hints for understanding the enormous evolutionary success of insects and also for understanding of functional design principles of large brains of mammals, including humans. Studies on alarm pheromone processing in the ant brain should provide an excellent case for this.

\section{REFERENCES}

Alaux, C., Sinha, S., Hasadsri, L., Hunt, G. J., Guzmán-Novoa, E., DeGrandiHoffman, G., Uribe-Rubio, J. L., Southey, B. R., Rodriguez-Zas, S., and Robinson, G. E. (2009). Honey bee aggression supports a link between gene regulation and behavioral evolution. Proc. Natl.Acad. Sci. U.S.A. 106, 15400-15405.

Billen, J. (1994)."Morphology of exocrine glands in social insects: an up-date 100 years after $\mathrm{Ch}$. Janet," in Les Insectes Sociaux, eds A. Lenoir, A. G. Arnold, and M. Lepage (Paris: Publications Universite Paris Nord), p. 214.

Blum, M. S. (1985). "Alarm pheromone." in Comparative Insect Physiology, Biochemistry and Pharmacology, Vol 9, eds G. A. Kerkut and L. I. Gilbert (Oxford: Pergamon) pp. 193-224.

Boeckh, J., and Ernst, K. D. (1987). Contribution of single unit analysis in insects to an understanding of olfactory function. J. Comp. Physiol. A. 161, 549-565.
Breed, M. D., Guzmán-Novoa, E., and Hunt, G. J. (2004). Defensive behavior of honey bees: organization, genetics, and comparisons with other bees. Annu. Rev. Entomol. 49, 271-298.

Brennan, P. A., and Kendrick, K. M. (2006). Mammalian social odours: attraction and individual recognition. Philos. Trans. R. Soc. Lond. B. Biol. Sci. 361, 2061-2078.

Brennan, P. A., and Zufall, F. (2006). Pheromonal communication in vertebrates. Nature 444, 308-315.

Chittka, L., and Niven, J. (2009). Are bigger brains better? Curr. Biol. 19, R995-R1008.

Couto, A., Alenius, M., and Dickson, B. J. (2005). Molecular, anatomical, and functional organization of the Drosophila olfactory system. Curr. Biol. 15, 1535-1547.

de Bruyne, M., Foster, K., and Carlson, J. R. (2001). Odor coding in the Drosophila antenna. Neuron 30, 537-552.

Dumpert,K.(1972).Alarmstoffrezenptoren auf der Antenne von Lasius fuliginosus

\section{ACKNOWLEDGMENTS}

This study was supported by grants from the Ministry of Education, Science, Culture, Sports and Technology of Japan (to Makoto Mizunami and Hiroshi Nishino) and Fyssen Foundation (to Nobuhiro Yamagata).

(Lart.) (Hymenoptera, Formicidae). Z. Vergl. Physiol. 76, 403-425.

Erber,J., Masuhr, T., and Menzel, R. (1980). Localization of short-term memory in the brain of the bee, Apis mellifera. Physiol. Entomol. 5, 343-358.

Fujiwara-Tsujii, N., Yamagata, N., Takeda, T., Mizunami, M., and Yamaoka, R. (2006). Behavioral responses to the alarm pheromone of the ant Camponotus obscuripes (Hymenoptera: Formicidae). Zool. Sci. 23, 353-358.

Galizia, C. G., and Rössler, W. (2010). Parallel olfactory systems in insects: anatomy and function. Annu. Rev. Entomol. 55, 399-420.

Galizia, C. G., Sachse, S., Rappert, A., and Menzel, R. (1999a). The glomerular code for odor representation is species specific in the honeybee Apis mellifera. Nat. Neurosci. 2, 473-478.

Galizia, C. G., Menzel, R., and Hölldobler, B. (1999b). Optical imaging of odorevoked glomerular activity patterns in the antennallobes of the ant Camponotus rufipes (Hymenoptera: Formicidae). Naturwissenschaften 86, 533-537.

Heimbeck, G., Bugnon, V., Gendre, N., Keller, A., and Stocker, R. F. (2001). A central neural circuit for experienceindependent olfactory and courtship behavior in Drosophila melanogaster. Proc. Natl. Acad. Sci. U.S.A. 98, 15336-15341.

Heisenberg, M. (2003). Mushroom body memoir: from maps to models. Nat. Rev. Neurosci. 4, 266-275.

Hildebrand, J. G., and Shepherd, G. M. (1997). Mechanisms of olfactory discrimination: converging evidence for common principles across phyla. Annu. Rev. Neurosci. 20, 595-631.

Hölldobler, B. (1995). The chemistry of social regulation: multicomponent signals in ant societies. Proc. Natl. Acad. Sci. U.S.A. 92, 19-22.

Hunt, G. J. (2007). Flight and fight: a comparative view of the neurophysiology and genetics of honey bee defensive behavior. J. Insect Physiol. 53, 399-410. 
Jefferis, G. S., Potter, C. J., Chan, A. M., Marin, E. C., Rohlfing, T., Maurer, C. R. Jr., and Luo, L. (2007). Comprehensive maps of Drosophila higher olfactory centers: spatially segregated fruit and pheromone representation. Cell 128, 1187-1203.

Joerges, J., Küttner, A., Galizia, C. G., and Menzel, R. (1997). Representations of odours and odour mixtures visualized in the honeybee brain. Nature 387, 285-288.

Kanzaki, R., Ikeda, A., and Shibuya, T. (1994). Morphological and physiological properties of pheromonetriggered flipfloping descending interneurons of the silkworm moth, Bombyx mori. J. Comp. Physiol. A. 175, 1-14.

Kanzaki, R., Soo, K., Seki, Y., and Wada, S. (2003). Projections to higher olfactory centers from subdivisions of the antennal lobe macroglomerular complex of the male silkmoth. Chem. Senses 28, 113-130.

Kirschner, S., Kleineidam, C. J., Zube, C., Rybak, J., Grünewald, B., and Rössler, W. (2006). Dual olfactory pathway in the honeybee, Apis mellifera. J. Comp. Neurol. 499, 933-952.

Kleineidam, C. J., Obermayer, M., Halbich, W., and Rössler, W. (2005). A macroglomerulus in the antennal lobe of leaf-cutting ant workers and its possible functional significance. Chem. Senses 30, 383-392.

Knaden, M., and Wehner, R. (2004). Path integration in desert ants controls aggressiveness. Science 305, 60.

Krofczik, S., Menzel, R., and Nawrot, M. P. (2008). Rapid odor processing in the honeybee antennal lobe network. Front. Comput. Neurosci. 2, 9.

Kuebler, L. S., Kelber, C., and Kleineidam, C. J. (2010). Distinct antennal lobe phenotypes in the leaf-cutting ant (Atta vollenweideri). J. Comp. Neurol. 51,352-365.

Li, Y. S., and Strausfeld, N. J. (1999). Multimodal efferent and recurrent neurons in the medial lobe of cockroach mushroom body. J. Comp. Neurol. 409, 647-663.

Menzel,R., and Giurfa,M.(2001).Cognitive architecture of a mini-brain, the honey bee. Trends Cogn. Sci. 5, 62-71.

Mizunami, M., Okada, R., Li, Y., and Strausfeld, N. J. (1998a). Mushroom bodies of the cockroach: the activity and identities of neurons recorded in freely moving animals. J. Comp. Neurol. 402, 501-519.

Mizunami, M., Weibrecht, J. M., and Strausfeld, N. J. (1998b). Mushroom bodies of the cockroach: their participation in place memory. J. Comp. Neurol. 402, 520-537.

Mizunami, M., Yokohari, F., and Takahata, M. (1999). Exploration into the adaptive design of the arthropod "microbrain." Zool. Sci. 16, 703-709.

Mizunami, M., Yokohari, F., and Takahata, M. (2004). Further exploration into the adaptive design of the arthropod "microbrain": I. Sensory and memory-processing systems. Zool. Sci. 21, 1141-1151.

Müller, D., Abel, R., Brandt, R., Zöcker, M., and Menzel, R. (2002). Differential parallel processing of olfactory information in the honeybee, Apis mellifera. J. Comp. Physiol. A. 188, 359-370.

Mysore, K., Subramanian, K. A., Sarasij, R. C., Suresh, A., Shyamala, B. V., VijayRaghavan, K., and Rodrigues, V. (2009). Caste and sex specific olfactory glomerular organization and brain architecture in two sympatric ant species, Camponotus sericeus and Camponotus compressus (Fabricius, 1798).Arthropod Struct. Dev. 38, 485-497.

Nakanishi, A., Nishino, H., Watanabe H., Yokohari, F., and Nishikawa, M. (2009). Sex-specific antennal sensory system in the ant Camponotus japonicus: structure and distribution of sensilla on the flagellum. Cell Tissue Res. 338, 79-97.

Nakanishi, A., Nishino, H., Watanabe, H., Yokohari, F., and Nishikawa, M. (2010). Sex-specific antennal sensory system in the ant Camponotus japonicus: glomerular organizations of antennal lobes. J. Comp. Neurol. 518, 2186-2201.

Nishikawa, M., Nishino, H., Misaka, Y., Kubota, M., Tsuji, E., Satoji, Y., Ozaki, M., and Yokohari, F. (2008). Sexual dimorphism in the antennal lobe of the ant Camponotus japonicus. Zool. Sci. 25, 195-204.

Nishino, H., Nishikawa, M., Mizunami, M., and Yokohari, F. (2009). Functional and topographic segregation of glomeruli revealed by local staining of antennal sensory neurons in the honeybee Apis mellifera. J. Comp. Neurol. 515, 161-180.

Nishino, H., Yamashita, S., Yamazaki, Y., Nishikawa, M., Yokohari, F., and Mizunami, M. (2003). Projection neurons originating from thermoand hygrosensory glomeruli in the antennal lobe of the cockroach. $J$. Comp. Neurol. 455, 40-55.

Okada, R., Ikeda, J., and Mizunami, M. (1999). Sensory responses and movement-related activities in extrinsic neurons of the cockroach mushroom bodies. J. Comp. Physiol. A. 185, 115-129.

Okada, R., Sakura, M., and Mizunami, M. (2003). Distribution of dendrites of descending neurons and its implications for the basic organization of the cockroach brain. J. Comp. Neurol. 458, 158-174.
Ozaki, M., Wada-Katsumata, A. Fujiwara, K., Iwasaki, M., Yokohari, F., Satoji, Y., Nishimura, T., and Yamaoka, R. (2005). Ant nestmate and non-nestmate discrimination by a chemosensory sensillum. Science 309, 311-314.

Rospars, J.P., and Hildebrand, J. G. (2000). Sexually dimorphic and isomorphic glomeruli in the antennal lobes of the sphinx moth Manduca sexta. Chem. Senses. 25, 119-129.

Sachse, S., Rappert, A., and Galizia, C. G. (1999). The spatial representation of chemical structures in the antennal lobe of honeybees: steps towards the olfactory code. Eur. J. Neurosci. 11, 3970-3982.

Sandoz, J., Deisig, N., de Brito Sanchez, M., and Giurfa, M. (2007). Understanding the logics of pheromone processing in the honeybee brain: from labeled-lines to across-fiber patterns. Front. Behav. Neurosci. 1, 5 .

Seki, Y., Aonuma, H., and Kanzaki, R. (2005). Pheromone processing center in the protocerebrum of Bombyx mori revealed by nitric oxide-induced anticGMP immunocytochemistry. J. Comp. Neurol. 481, 340-351.

Stopfer, M., Bhagavan, S., Smith, B. H., and Laurent, G. (1997). Impaired odour discrimination on desynchronization of odour-encoding neural assemblies. Nature 390, 70-74.

Strausfeld, N. J. (1976). Atlas of an Insect Brain. Berlin: Springer-Verlag.

Suh, G. S., Wong, A. M., Hergarden, A. C., Wang, J. W., Simon, A. F., Benzer, S., Axel, R., and Anderson, D. J. (2004). A single population of olfactory sensory neurons mediates an innate avoidance behaviour in Drosophila. Nature 431, 854-859.

Vander Meer, R. K. and Alonso, L. E. (1998). "Pheromone directed behavior in ants," in Pheromone Communication in Social Insects, eds R. K. Vander Meer, M. D. Breed, M. L. Winston, and K. E. Espelie (Oxford: Westview Press), pp. 159-192.

Vareschi,E. (1971).Duftunterscheidung bei den Honigbienen-Einzelzellableitungen und Verhaltensreaktionen. Z. Vergl. Physiol. 75, 143-173.

Vosshall, L. B., and Stocker, R. F. (2007). Molecular architecture of smell and taste in Drosophila. Annu. Rev. Neurosci. 30, 505-533.

Wang, L., and Anderson, D. J. (2010). Identification of an aggressionpromoting pheromone and its receptor neurons in Drosophila. Nature 463, 227-231.

Watanabe, H., Nishino, H., Nishikawa, M. Mizunami,M., and Yokohari, F. (2010) Complete mapping of glomeruli based on sensory nerve branching pattern in the primary olfactory center of the cockroach Periplaneta americana. J. Comp. Neurol. (in press)

Yamagata, N., Fujiwara, N., Yamaoka, R., and Mizunami, M. (2005). Pheromone communication and the mushroom body of the ant, Camponotusobscuripes (Hymenoptera: Formicidae). Naturwissenschaften 92, 532-536.

Yamagata, N., and Mizunami, M. (2010). Spatial representation of alarm pheromone information in a secondary olfactory center in the ant brain. Proc. R. Soc. B. (in press).

Yamagata, N., Nishino, H., and Mizunami, M. (2006). Pheromone-sensitive glomeruli in the primary olfactory centre of ants. Proc. R. Soc. B. 273 , 2219-2225.

Yamagata, N., Nishino, H., and Mizunami, M. (2007). Neural pathways for the processing of alarm pheromone in the ant brain. J. Comp. Neurol. 505, 424-442.

Yamagata, N., Schmuker, M., Szyszka, P., Mizunami, M., and Menzel, R. (2009). Differential odor processing in two olfactory pathways in the honeybee. Front. Syst. Neurosci. 3:16. doi: 10.3389/neuro.06.016.2009.

Yurkovic, A., Wang, O., Basu, A. C., and Kravitz, E. A. (2006). Learning and memory associated with aggression in Drosophila melanogaster. Proc. Natl. Acad. Sci. U.S.A. 103, 17519-17524.

Zube, C., Kleineidam, C. J., Kirschner, S., Neef, J., and Rössler, W. (2008). Organization of the olfactory pathway and odor processing in the antennal lobe of the ant Camponotus floridanus. J. Comp. Neurol. 506, 425-441.

Zube, C., and Rössler, W. (2008). Casteand sex-specific adaptations within the olfactory pathway in the brain of the ant Camponotusfloridanus. Arthropod. Struct. Dev. 37, 469-479.

Conflict of Interest Statement: The authors declare that the research was conducted in the absence of any commercial or financial relationships that could be construed as a potential conflict of interest.

Received: 27 February 2010; paper pending published: 24 March 2010; accepted: 10 May 2010; published online: 08 June 2010

Citation: Mizunami M, Yamagata N and Nishino H (2010) Alarm pheromone processing in the ant brain: an evolutionary perspective. Front. Behav. Neurosci. 4:28. doi: 10.3389/fnbeh.2010.00028

Copyright $\odot 2010$ Mizunami, Yamagata and Nishino. This is an open-access article subject to an exclusive license agreement between the authors and the Frontiers Research Foundation, which permits unrestricted use, distribution, and reproduction in any medium, provided the original authors and source are credited. 